\title{
Addressing the emergence of plurality: Towards a global web of local design narratives.
}

\author{
Heitor Alvelos ${ }^{a *}$, Susana Barretoa \\ alD+/Unexpected Media Lab, University of Porto \\ *halvelos@fba.up.pt
}

\begin{abstract}
:
The present research addresses empirical evidence of a predominantly westerncentric, canonical narrative in Design: it argues that Design Education and Research may benefit from substantial expansion and diversification upon this traditionally prevalent narrative, in line with a range of disciplines currently addressing equivalent challenges. Furthermore, we posit that a plurality in Design may begin by addressing its multiple narratives at a local level, upon which a global web of detailed knowledge may emerge: complex, global discourses may require articulations of the intrinsically contextual. With this premise in mind, preliminary research has been held in Porto, Portugal, from 2018 to 2021, through in-person interviews with retired Design teachers and professionals; these have locally confirmed the above hypothesis, namely in regards to issues of gender, and ties with local crafts and industry. We now aim at reverting these findings into higher education curricula, and national and international scalability of enquiry.
\end{abstract}

Keywords: Local design; Education networks; Design studies; Design Decolonisation

\section{Introduction}

Design faces a particular crossroads at this point in time: while curricular configuration of the discipline has occurred primarily via a fairly linear narrative that has been predominantly male and Western-centric, recent multi-disciplinary shifts have been addressing a pressing need to reconsider prevailing narratives and corresponding roles, references, and assumptions in public discourse. A series of factors have been contributing to this renewed sense of a need for plurality: a global permeability to multicultural systems, as well as the increasing relevance of post-colonialist studies and an overall heightened awareness of race and gender issues with corresponding socio-political 
dynamics of dominance and visibility. All of these are largely traversing younger generations whose default relationship with knowledge is often in itself based on empirical multi-perspectivity.

In parallel, we must acknowledge that Design itself has broadened its scope considerably in the past decades, expanding from an original range of aesthetic and functional parameters into strategic, perceptive and ideological territories: in truth, a range of activities and outputs that might, in past instances, have been relegated to fairly indiscriminate categories of traditional craft, improvisational tools or amateur communication processes, are now undergoing a process of re-appreciation as innovative Design objects and activities - a rather urgent, if somewhat paradoxical, process if one considers the wealth of unwritten knowledge and aesthetic rupture thereby contained.

With this is mind, the present research argues for the need to reconsider established content in Design narratives and outputs; we further argue for the key role of Design Education in their identification and dissemination, proposing that higher education institutions are particularly wellsuited to conducting enquiry on a contextual level pertaining to their own individual ecosystem. By looking into Portuguese Design as a case study, the research aims at unearthing a detailed reconstruction of local Design narratives that legitimises a range of authors, processes and specimens that remain not just largely unacknowledged in their own locus, they remain overshadowed by a largely imported narrative when it comes to the dissemination of said narratives. From here, the research posits that similar occurrences might be taking place in further contexts, thus proposing an international web of enquiry into local Design narratives.

\section{Research question}

Given the emerging awareness of (and engagement with) the need for historical, social and disciplinary decolonisation in contemporaneity, with consequent cultural, political and epistemological impact, how can Design narratives ensure their own exponential process of plurality and multiple perspectivity, in face of traditionally reductive content?

\section{Context}

In recent years, Design has begun to embrace multiple perspectivity, a clearer understanding that History weaves complementary and contrasting versions of a shared narrative. The need to embrace Design's multiple sites runs concurrently with a renewed awareness of complex socio-cultural imbalances, both contemporary and historical, with impact traversing a multitude of disciplines.

Margolin (1996) argued that "there can be no single Design history." And yet, as pointed out by Triggs (2011:3), a History of Design has largely been recounted from an essentially male dominant perspective. As pointed out by Adamson et al (2011), we need "[an] ethical history of what Design should do". The present research is in the process of gathering a body of knowledge in the context of Portuguese Design, and argues that a network dedicated to equivalent processes of local scrutiny may be an effective approach towards the aforementioned multiple perspectivity. We further vouch for higher education as a particularly effective context for both the study and the dissemination of new knowledge in the field.

Within the context of the present research, empirical evidence gathered from fifty one interviews with Portuguese retired Art and Design academics has confirmed a predominance of western-centric 
narrative in design. Concurrently, Design students involved in research workshops have themselves pointed out how references provided in courses tend to be invariably foreign. A range of factors explain this trend: a central role is played by Design values and history taught through a European and American canon of largely male designers that seem to have set an aesthetic and narrative foundation, and a dominance of mainstream discourse has largely obliterated the work produced by non-Western cultures outside the modernist paradigm of universal design, such as crafts.

As noted by Ansari (2018), the starting point is "challenging and critiquing the current status quo in mainstream contemporary academic and professional discourse and bringing greater depth to the conversations happening around issues of gender, race, culture, and class." A plurality in Portuguese Design Education thus aims to be at the forefront of current innovation policies: one of the strategic actions by the European Design Leadership Board (2014) positions Design Research for the 21st Century, and the recent New European Bauhaus initiative (2000) calls for participatory Design as key to development and inclusion.

Nevertheless, it must be pointed out that this research does not aim at de-legitimising existing canons; instead, it means to place them as components of a broader, node-based geometry, as opposed to maintaining a "center-periphery" model that has dominated discourse in the past.

Portugal has become a natural case study and testbed for the above ambitions due to the authors' own experience of decades as teachers and researchers in Portuguese Design Education institutions. The male- and foreign-dominated narrative has been an evidence throughout: Design as a successful and canonical practice is fundamentally taught at undergraduate level as happening elsewhere. Other occurrences are in themselves telling of an imbalance: despite a predominance of female students, teaching staff is systematically, predominantly male, as are the few national references that have managed to rise to inclusion onto a broader narrative - as noted by Aguiar (M. Aguiar, personal communication, June 15, 2019), the first woman teacher at the Porto School of Fine Arts in 1976, the gender asymmetry was striking, with an average of $75 \%$ of female students contrasting with an entirely male faculty. As such, gender is inevitably one of the core issues to be addressed in a plural Design narrative, and works as a particularly expressive example of the challenges at stake.

Costa (2019) states that until the early 20th Century there is scarce evidence in Portugal of a participation of women in Design practice, and this continued until a surge in the 1960s; we posit that this invisibility may result from traditional gender role paradigms, certainly reinforced throughout the former conservative regime, ended in 1974. However, a late inscription of Design as such in the Portuguese context is observable: primarily a side commercial practice by artists venturing into graphic arts and typography, for decades Design occupied a subsidiary role to the fine arts.

A turning point in the country's political and social fabric, the Revolution of 1974 triggered a surge of written and graphic expression celebrating free speech and civic engagement. Publications, associations, gatherings, murals, events, posters: largely driven by the spur of the moment, the abundance of graphic specimens that followed the revolution is largely preserved at the Ephemera Archives (Pereira, 2021), mostly awaiting scrutiny. This body of evidence records the shifting visual languages of a nation, and yet, most specimens are of uncertain authorship, and can only be traced back through testimony and snowballing.

We further posit that a multi-ethnic insight is largely overdue in the case of Portugal: following the 1974 revolution and the subsequent concession of sovereignty to Portugal's African colonies, a surge in immigration translated in the appearance of self-organised suburban communities of African provenance. To this day, these communities largely retain a considerable degree of social stigma that 
only now begins to take tentative steps towards actual visibility and contributions to socio-political dialogue. Similarly, customs and practices of these communities as contributions to national cultural and creative narratives and dynamics remain residual. We thus propose to add to a belated reconstruction of this legacy towards its rightful place in the national narrative of cultural artefacts.

\section{Methodologies}

Two prior research endeavours undertaken by the authors between 2018 and early 2021 have unearthed detailed, contextual ties to Portuguese Design:

- A wealth of testimonies by retired designers pertaining to a first-person recount of Art and Design Schools in the 1970s and 1980s; intricate memories of Design production were recalled, where authors and protagonists had often been forgotten, or even self-excluded (Wisdom Transfer: towards the scientific inscription of individual legacies in contexts of retirement from art and design higher education and research);

- Strategic and creative mediation with local crafts associations and small industries currently facing viability challenges; Design Research was essentially employed as a facilitator of self-empowerment (Anti-Amnesia: design research as an agent for narrative and material regeneration and reinvention of vanishing Portuguese manufacturing cultures and techniques).

The research adopted mixed methods, combining pre-existing statistical data and observational analysis. Preliminary statistical data was central to the establishment of a chronology and the identification of journey maps, which later helped single out key players to be interviewed. From then onwards, studies followed a snowball sampling technique, in which the initial study subjects recruited future subjects from among their acquaintances. Ethnographic interviewing subscribed to Atkinson et al's (2001) premise that it helps gather rich, detailed data directly from interviewees, providing tangible insights into the ways participants recollected their experience; this in turn has helped to fine-tune ensuing interviews.

Images of visual artefacts such as books, letters, photographs, paintings, and magazine cut-outs, were unearthed and documented during these interviews. The ethnographic content and information gathered was interwoven and analysed through timeline maps, field notes, personal inventories, diary studies, factual and archival data: this led to categorization by patterns of gender, age group, degree and professional path.

With local crafts associations and small industrial factories, interviews were complemented with a range of pedagogical experiments involving the hybridization of outputs by crossing source materials, aesthetics and content. With former academics, interviews gave way to workshops involving mediated ties and briefings with current students, as well as a series of moderated public sessions. From these actions, further thematic networks were revealed. Additionally, the unearthed content has become the source material for a series of public exhibitions, a Summer School, and a projected archive in cooperation with the Arts Library at the University of Porto.

\section{Analysis}


Pursued under a networked perspective, the specific ties currently under development with eleven Portuguese Design Schools and local narratives/agents are naturally conductive towards a logic of simultaneous contrast and complementarity: Schools may offer a positively differentiated curricular content, and may resort to one another's competences and resources when needed and appropriate. Furthermore, the contrast/complementarity logic may become a motto for researchrelated dynamics: the Schools identified in Figure 1 have congregated their Design Research competences in a shared Research Center (ID+), where critical mass attains further density and scale while fostering tangible opportunities for complementary know-how. We argue that this model of cooperation is scalable, and may therefore ultimately come to represent the aforementioned global

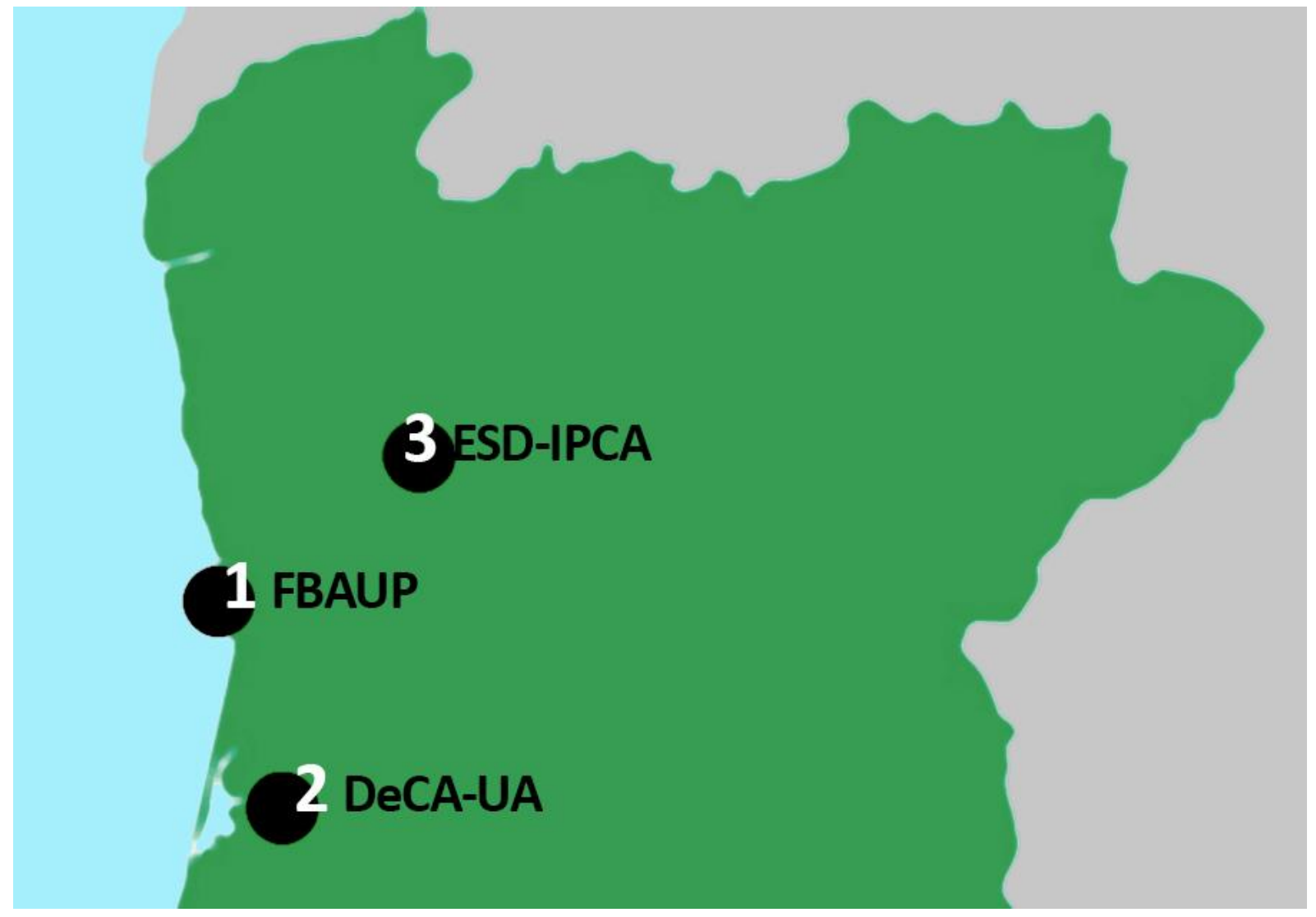

network.

Figure 1. A map of the Northern region of Portugal, outlining three Design Schools that converge in a joint research center and PhD program. 1. School of Arts of the University of Porto: Design BA created in 1974; strong ties with Fine Arts, Engineering and cultural agents; recent investment in R\&D with start-ups. 2. Communication and Art Department, University of Aveiro: Design BA created in 1995; strong ties with local industry, namely ceramics, shoemaking and fishing. 3. Higher School of Design, Polytechnic Institute of Cávado and Ave: Design BA created in 2015; strong ties with local industry, namely furniture and shoes; primarily market and job-oriented education. Image by the authors.

As an example of tangible dynamics at work, the aforementioned Anti-Amnesia has congregated researchers from Schools 1, 2 and 3, while Wisdom Transfer has congregated researchers from Schools 1 and 3, as well as researchers from three other Design Schools in the region. Furthermore, as an example of educational cooperation, a joint $\mathrm{PhD}$ program is offered in cooperation between Schools 1 and 2.

These various instances of validation bode well for further, stable curricular insertion of detailed recounts by Design professionals, and a strengthening of reciprocal knowledge between a Design School and its immediate ecosystem. We posit that this may become a duty of Design Schools, and 
that this reciprocal dynamic may be replicated/"fractalized" and customised according to further local, regional and national contexts, pointing towards a shared endeavour on an international scale. This approach, we argue, may contribute to the aforementioned plurality in Design Education by ensuring that the inscription and relevance of each Design narrative is attained through close, rooted scrutiny. Contextual narratives can subsequently circulate as needed for pedagogical ends, among a wealth of knowledge that is rooted locally but can travel globally.

\section{Conclusions and future developments}

The above actions and findings point towards a series of advantages in fostering plurality in Design narratives and education:

- The current, multidisciplinary discussion on the decolonising of culture and society, as well as its concern over formerly fixed models of historical narrative, may greatly benefit from the contributions of Design;

- reciprocally, Design must continue its own endeavour towards plurality, namely by employing higher education and curricular content as prime tools for dissemination of multi-perspectivity;

- a purposeful differentiation among Design Schools according to their local and regional narratives may lead to the exponential enrichment of Design Education through the consolidation of a complementary web of empirical, contextual knowledge;

- additionally, the above differentiation may be obtained by strengthening working ties with local agents, particularly crafts and industry, through curricular content and activity; preliminary research on this front has revealed promising reciprocal gains;

- as a local/regional approach, the aforementioned contrast-complementarity model may be rehearsed as an international web of Design Schools engaging with their respective local partners and narratives, while strengthening complementary cooperation within the proposed Design School Network.

- this particular objective is a longer-term endeavour, and further preliminary research is needed in order to gradually establish a preliminary network of Design Schools. At this stage, the research is looking into how eleven Portuguese Schools may build upon this proposed model of reciprocal contrast-complementarity;

- an international network is the logical next step. A dialogue in this respect is welcome by the authors, particularly within the framework of the New European Bauhaus (NEB): its motto and the strategic scope of the present research clearly share an ethos of "bottom up" re-evaluation and participation. We therefore invite Design delegates to engage with us in a proposal for a NEBassociated network.

\section{References}

Adamson, G., Riello, G, \& Teasley (2011). Global Design History. London: Taylor \& Francis.

Alvelos, H. (2019). Unexpected Media Lab. Porto: ID+/University of Porto. 
Alvelos, H., \& Chatterjee, A. (2021). Anti-Amnesia. Retrieved 19 May, 2021, from

http://antiamnesia.fba.up.pt

Alvelos, H., Chatterjee, A., \& De Almeida, P. C. (2021). Anti-Amnesia: design research as an agent for narrative and material regeneration and reinvention of vanishing Portuguese manufacturing cultures and techniques. Porto: ID+/Unexpected Media Lab.

Ansari, A. (2018, March 8-10). What a Decolonisation of Design Involves: Two Programmes for Emancipation [Paper presentation]. Beyond Change: Questioning the role of Design in times of global transformations, Basel, Switzerland.

Atkinson, P., Coffey, A., Delamont, S., Lofland, J., \& Lofland, L. (Eds.). (2001). Handbook of Ethnography. New York: SAGE Publications.

Barreto, S., Lima, C. \& Penedos-Santiago, E. (Eds.) (2021). Wisdom Transfer. Porto: ID+/Unexpected Media Lab.

Barreto, S., Lima, C. \& Penedos-Santiago, E. (2021). Wisdom Transfer (Website). Retrieved 19 May, 2021, from http://wisdomtransfer.fba.up.pt

Costa, J. S. B. (2019, October). The discourses of graphic Design in its emergence in Portugal (1960 2000) (PhD Thesis). Porto: University of Porto School of Fine Arts.

European Design Leadership Board, Koskinen, T., \& Thomson, M. (2014, June). Design for growth \& prosperity. Brussels: EU publications.

Margolin, V. (1996). Teaching Design History. Statements, 11(2).

New European Bauhaus. (2021, January 18). Our conversations will shape our tomorrow. https://europa.eu/new-european-bauhaus/index_en

Pereira, J. P. (2021, May 3). Ephemera Archives. Retrieved 19 May, 2021, from

https://ephemerajpp.com/

Triggs, T. (2011). Graphic Design History: Past, Present, and Future. Design Issues 17(1), 3-6.

Author Bios:

\section{Heitor Alvelos}

PhD Design (RCA, 2003); MFA (SAIC, 1992). Associate Professor at the University of Porto, where he directs the PhD Design and the Unexpected Media Lab / ID+ Research Center. Current Chairman of the Scientific Board (HSS), Foundation for Science and Technology.

\section{Susana Barreto}

Design researcher and educator in design at the University of Porto. Interests converge in the role of ethics in visual communication, design and crime, design culture, visual methodologies and life stories of Portuguese designers.

\section{Acknowledgements:}

The authors wish to thank the Foundation for Science and Technology for the successful funding bids of ID+, Wisdom Transfer and Anti-Amnesia. Further thanks to all AntiAmnesia researchers, particularly Eliana Penedos-Santiago and Cláudia Raquel Lima, all individuals interviewed, and all students and teachers involved in the associated pedagogical activities. 